\title{
EFECTO DE BIOFERTILIZANTE Y MATERIA ORGÁNICA EN EL RENDIMIENTO DE CLONES DE JOJOBA Sinmondsia chinesis(Link) Scheneider EN LOS PICHONES TACNA 2014
}

\author{
BIOFERTILIZER EFFECT AND ORGANIC MATTER IN THE \\ PERFORMANCE OF CLONES OF JOJOBA Sinmondsia chinesis (Link) Schneider \\ TACNA PIGEONS IN 2014
}

${ }^{1}$ Nelly Arévalo Solsol

RESUMEN

El objetivo de la presente investigación fue determinar el efecto de la aplicación de biofertilizante y materia orgánica en las características morfológicas y el rendimiento de clones de jojoba Sinmondsia chinensis (Link) Scheneider. Se empleó el diseño experimental de DBCA con 5 tratamientos y 4 repeticiones. El experimento se condujo en el fundo Los Pichones ubicada a $550 \mathrm{msnm}$. Los tratamientos fueron: $T 1=$ bioferilizante $+0,5 \mathrm{~kg} /$ planta M.O, T2=biofertilizante $+1,0 \mathrm{~kg} /$ planta M.O, T3= biofertilizante $+1,5 \mathrm{~kg} /$ planta M.O, T4= biofertilizante $+2,0 \mathrm{~kg} /$ planta M.O y T5 = biofertilizante +2,5 kg/planta M.O. Los resultados indican que la aplicación de biofertilizante y materia orgánica tienen efectos positivos en las caracteristicas morfológicas y el rendimiento de clones de jojoba. Los caracteres altura de planta, número de frutos se modificaron con la aplicación de biofertilizante y materia orgánica. Los caracteres forma de hoja, de frutos y posición de frutos no se modificaron con la aplicación de biofertilizante y materia orgánica. Con la aplicación de 2,5 kg/planta de materia orgánica más biofertilizante se obtuvo un peso de semilla de $784,40 \mathrm{~g} /$ planta superando al testigo que tuvo un rendimiento de $393,40 \mathrm{~g} / \mathrm{planta}$. Estos resultados son considerados como una buena alternativa para mejorar los suelos y mantener una producción orgánica sostenible en lajojoba.

Palabras clave: Jojoba, clon, biofertilizante, materia orgánica y rendimiento

\section{ABSTRACT}

The aim of this investigation was to determine the effect of the application of biofertilizer and organic matter in the morphological characteristics and performance of jojoba clones Sinmondsia chinensis (Link) Schneider. DBCA experimental design with 5 treatments and 4 replications was used. The experiment was conducted at the farm The Pigeons located 550 meters. The treatments were: $T 1=$ biofertilizer $+0,5 \mathrm{~kg} /$ plant $\mathrm{MO}+, \mathrm{T} 2=$ biofertilizer $+1,0 \mathrm{~kg} /$ plant MO, T3 = biofertilizer $+1,5 \mathrm{~kg} /$ plant MO, T4 = biofertilizer $+2,0 \mathrm{~kg} / \mathrm{plant} \mathrm{MO}$ and $\mathrm{T} 5=$ biofertilizer $+2,5 \mathrm{~kg} / \mathrm{plant} \mathrm{MO}$. The results indicate that application of biofertilizer and organic matter have positive effects on the morphological characteristics and performance of jojoba clones, characters plant height, number of fruits were modified with the application of bio-fertilizer and organic matter. The leaf shape, fruit and fruit character position did not change with the application of biofertilizer and organic matter. With the application of $2,5 \mathrm{~kg} / \mathrm{p}$ lant organic matter more biofertilizer seed weight 784,40 g/plant was obtained by beating the witness who had a yield of $393,40 \mathrm{~g} /$ plant. These results are considered as a good alternative to improve soil and maintain a sustainable organic production of jojoba

Keywords: Jojoba, clone, bio fertilizer, organic matter and performance

\section{INTRODUCCIÓN}

La jojoba Sinmondsia chinensis (Link) Scheneider, planta perenne, dioica, de uno a cuatro metros de altura, es el único vegetal que produce cera líquida. El aceite se extrae por prensado de las semillas y se utiliza principalmente en productos cosméticos, mezclado con otras sustancias o simplemente sólo; despertó un gran interés mundial debido a las múltiples aplicaciones que de ellas se obtienen; siendo además un recurso renovable y amigable con el medio ambiente a diferencia del petróleo.

La jojoba se presenta como una alternativa de cultivo en la costa norte y sur del país, debido a su gran adaptación a las condiciones climáticas y edáficas de la zona; la rusticidad que se manifiesta a condiciones de sequía y salinidad, asi como su resistencia a plagas y enfermedades.

La agricultura orgánica es un sistema productivo que propone evitar e incluso excluir totalmente los fertilizantes y pesticidas sintéticos en la producción agrícola; en lo posible reemplaza las fuentes externas tales como sustancias quími-

'Doctora en Ciencias Ambientales, Ingeniera Agrónoma. Docente Principal de la Facultad de Ciencias Agropecuarias de la Universidad Nacional Jorge Basadre Grohmann. Tacna-Perú

*Esta investigación contó con la colaboración de la Dra. Rosario Zegarra Zegarra. 
Arévalo, N. Efecto de biofertilizante y materia orgánica en el rendimiento de clones de jojoba en los Pichones Tacna 2014.

cas y combustibles adquiridos comercialmente por recursos que se obtienen dentro del mismo predio o en sus alrededores; dichos recursos internos incluyen la energía solar y eóli$\mathrm{ca}$, el control biológico de las plagas, el nitrógeno fijado biológicamente y otros nutrientes que se liberan a partir de la materia orgánica o los recursos del suelo. Las opciones que fundamentan la agricultura orgánica son la máxima utilización de la rotación de cultivos, rastrojos vegetales, abono animal, leguminosas, abonos verde, desechos orgánicos, rocas fosfóricas, control biológico de plagas con miras a mantener la fertilidad del suelo y sus estructuras, suministro de nutrientes vegetales y el control de los insectos, malezas y otras plagas.

Arévalo y Fernández (2011) evaluaron clones de jojoba durante cuatro años, reportan que los clones de mejor rendimiento promedio fueron C1-11 con $820,80 \mathrm{~g}$, seguido del clon C5-46 con 545,60 g y en tercer lugar el clon C2-21 con $411,75 \mathrm{~g}$.

Con la aplicación de biofertilizantes más materia orgánica en clones de jojoba se pretendió incrementar los rendimientos y mejorar la fertilidad de los suelos. Es necesario aplicar tecnologías adecuadas buscando productividad y sostenibilidad del cultivo en las condiciones áridas salinas de Tacna.

El objetivo del estudio fue comparar el efecto de biofertilizante y la materia orgánica en las características morfológicas y en el rendimiento de clones de jojoba en el fundo Los Pichones, Tacna-2014.

\section{MATERIALES Y MÉTODOS}

\section{Tipoy diseño de la investigación}

El tipo y el diseño de investigación fue experimental ya que se emplearon las variables independientes (materia orgánica y biofertilizantes) para analizar los efectos en las variables dependientes como el rendimiento y las características morfológicas en clones de jojoba.

El trabajo de investigación se realizó en el fundo Los Pichones de propiedad de la Facultad de Ciencias Agropecuarias de la Universidad Nacional Jorge Basadre Grohmann de Tacna, siendo las coordenadas geográficas: Latitud Sur $17^{\circ} 59^{\prime} 38^{\prime \prime}$; Longitud Oeste $74^{\circ} 14^{\prime} 22^{\prime \prime}$; Altitud 550 m.s.n.m.

\section{Diseño experimental}

Se emplé el diseño experimental de Bloques Completos al Azar (DBCA). Se tuvo 5 tratamientos y 4 repeticiones y los tratamientos fueron los siguientes:

T $1=$ bioferilizante $+0,5 \mathrm{~kg} / \mathrm{planta} \mathrm{M} . \mathrm{O}$, $\mathrm{T} 2=$ biofertilizante $+1,0 \mathrm{~kg} /$ planta M.O,

T3= biofertilizante $+1,5 \mathrm{~kg} /$ planta M.O, T4=biofertilizante $+2,0 \mathrm{~kg} /$ planta M.Oy

$\mathrm{T} 5=$ biofertilizante $+2,5 \mathrm{~kg} /$ planta M.O.

\section{Preparación de la materia orgánica (compost)}

El compost es un abono orgánico que resulta de la transformación de la mezcla de residuos orgánicos de origen vegetal y animal que han sido descompuestos bajo condiciones controladas, para ello se utilizó estiércol de ganado vacuno más rastrojos de cosechas (tomate, pimiento, brócoli, orégano, tara y otros). La característica química del compost depende de la cantidad y tipo de insumos utilizados, asi como las condiciones ambientales que dominaron durante el proceso de descomposición, realizándose la labor de "volteo" para mezclar los componentes y homogenizar aplicando agua para inducir a la fermentación.

El proceso de la obtención del compost duró 3 meses.

\section{Aplicación de la materia orgánica}

El estićrcol de ganado vacuno previamente compostado se aplicó antes del trasplante en cantidades según los tratamientos. El biofertilizante se aplicó en el suelo en la zona de la rizósfera a una concentración de $0,5 \mathrm{~kg} / \mathrm{ha}$ en dos momentos al inicio de la floración femenina y al llenado de los frutos.

\section{Población y muestra}

El experimento se condujo en clones instaladas de seis años de edad y la selección de las plantas fue al azar, la cual se seleccionó de una población de 100 plantas; se tomaron al azar 4 plantas/tratamiento, las cuales fueron marcadas para aplicar la materia orgánica (figura 1, figura 2, figura 3 y figura 4 respectivamente).

Cosecha. Se realizó la cosecha de forma manual de acuerdo a la maduración de los clones. Seguidamente se procedió a la limpieza y clasificación de las semillas para su conteo y pesado respectivo.

\section{Características evaluadas}

\section{Características morfológicas}

Se realizó la caracterización morfológica de 54 plantas de la población. La técnica utilizada fue la observación visual. Asimismo el instrumento de la recolección de los datos fue la medición manual, teniendo en cuenta las siguientes evaluaciones:

Altura de plantas $(\mathrm{cm})$ : para la evaluación de esta variable se consideró medir desde el cuello de la planta hasta el brote de mayor altura, realizando esta evaluación con ayuda de una regla graduada, asimismo se realizó la evaluación de: posición de frutos, diámetro de follaje, forma de fruto, arquitectura de planta entre los caracteres cualitativos y numero de frutos con carácter cuantitativo.

\section{Evaluación del rendimiento}

$\mathrm{El}$ instrumento de la recolección de los datos fue el conteo manual e instrumental utilizando una balanza. La metodologia aplicada para la recolección de datos fue el muestreo aleatorio simple. Se marcaron 4 plantas por tratamiento y se realizaron las siguientes observaciones: Peso de semillas (g), con la ayuda de una balanza digital se pesaron las semillas por tratamiento.

Número de semillas, se contó el total de semillas por tratamiento.

\section{Procesamiento y análisis de datos}

Las pruebas estadisticas utilizadas fueron ANDEVA a un nivel de significación del $5 \%(\mathrm{p}=0,05)$. Para determinar 


\section{Ciencia \& Desarrollo}

Arévalo, N. Efecto de biofertilizante y materia orgánica en el rendimiento de clones de jojoba en los Pichones Tacna 2014.

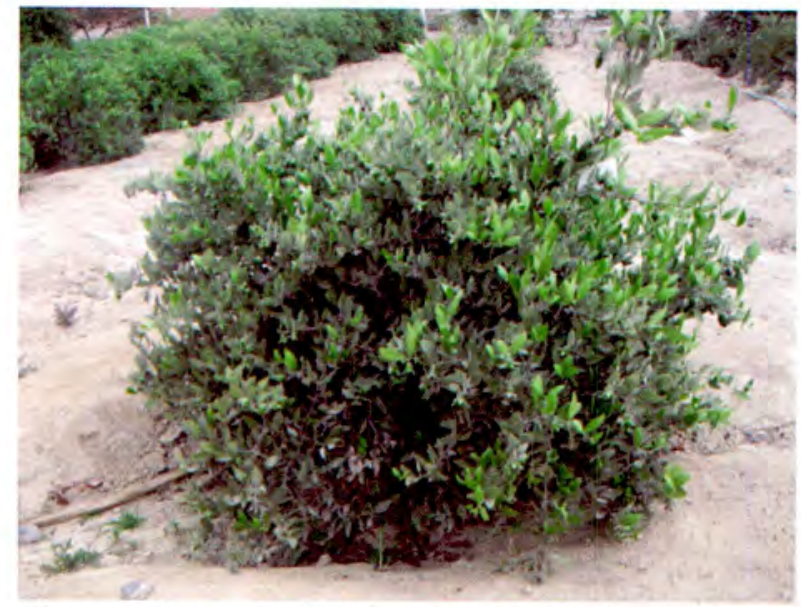

Figura 1. Planta seleccionada para aplicación de tratamientos.

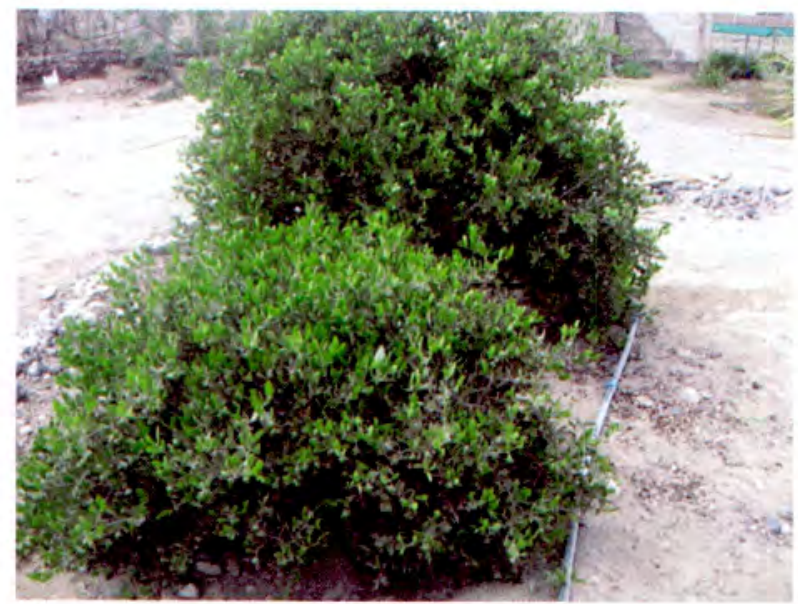

Figura 3. Plantas seleccionadas para aplicación de tratamientos.

las diferencias de los tratamientos se utilizó la prueba de significación de Duncan a un nivel de significación del $5 \%$. Para estas pruebas se utilizó el paquete estadístico Infostat Versión Estudiantil.

\section{RESULTADOS}

\section{Características morfológicas}

Evaluación morfológica de clones de jojoba. Los Pichones -Tacna 2014. (Ver tabla 2).

Posición de frutos (ver figura 5, 6 y 7 ).

Forma de frutos (ver figura 8).

En cuanto a los caracteres morfológicos los clones presentan una variabilidad genética en cuanto a forma y tamaño de hojas y frutos. Los clones presentan una posición de frutos de forma continua (figura 6 y 7 ), cuando encontramos un fruto en cada nudo de ramillas y de tipo alterno cuando encontramos un fruto en forma alterna (figura 5). La mayoría de los clones presentan una arquitectura de planta de buena a muy buena arquitectura de planta que son las más

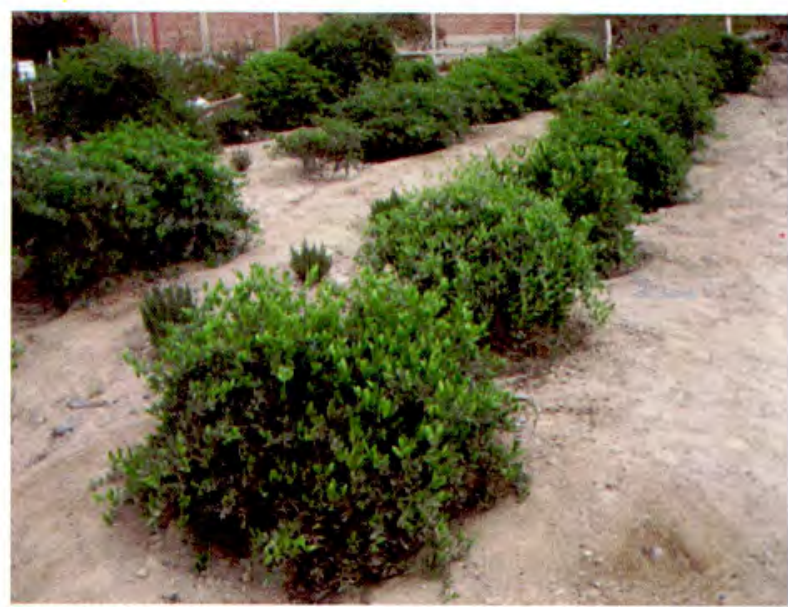

Figura 2. Plantas seleccionadas para aplicación de tratamientos.

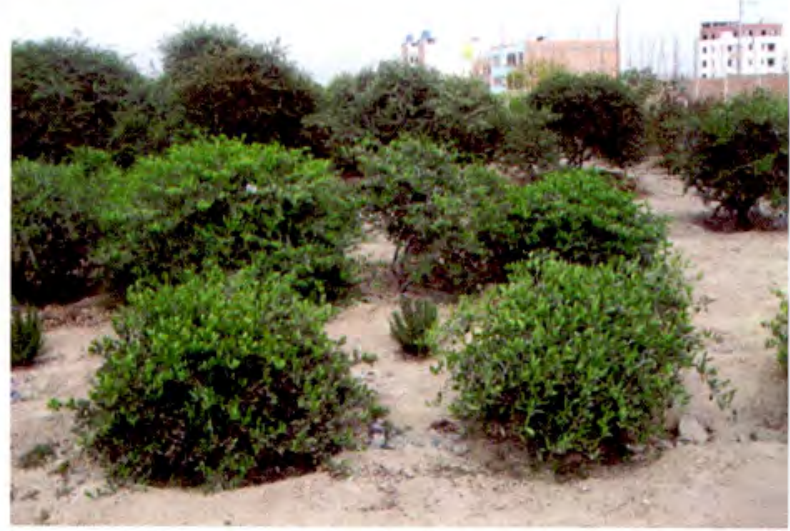

Figura 4. Vista panorámica de plantas seleccionadas.

deseables agronómicamente.

\section{Evaluación de Rendimiento}

Tabla 1. Análisis de variancia del peso (g) de clones de jojoba con aplicación de biofertilizantes y materia orgánica. Los Pichones-Tacna 2014.

\begin{tabular}{cccccc}
\hline $\begin{array}{c}\text { Fuentes de } \\
\text { variancia }\end{array}$ & GL & S C & C M & FC & Ft \\
\hline Repetición & 4 & 5166,40 & 1291,60 & 0,24 & 5,6 \\
Tratamiento & 4 & 579569,60 & 144892,45410, & $26,78^{*}$ & 5,6 \\
Error & 16 & 86560,00 & 00 & & \\
Total & 24 & 671296,00 & & & \\
\hline
\end{tabular}

CV $12,03 \%$

*Nivel de significación $5 \%$

El análisis de variancia del peso $(\mathrm{g})$ nos indica que para las repeticiones $\left(\mathrm{Fc}=0,24, \mathrm{GL}={ }_{4,16} ; \mathrm{Ft}_{0,05} \mathrm{GL}_{4,16=}, 5,64\right)$ no se encontraron diferencias significativas. En lo que respecta a tratamientos evaluados presentan diferencias significativas $\left(\mathrm{Fc}=26,78 \mathrm{GL}_{4,16} ; \mathrm{Ft}_{0,05} \mathrm{GL}_{4,16}=5,64\right)$ entre los tratamientos con aplicación de biofertilizante y materia orgánica. Para detectar las diferencias entre los tratamientos debe realizarse la prueba de significación de Duncan (tabla 3). 
Arévalo, N. Efecto de biofertilizante y materia orgánica en el rendimiento de clones de jojoba en los Pichones Tacna 2014.

Tabla 2. Características morfológicas de clones de jojoba Los Pichones Tacna-2014.

\begin{tabular}{|c|c|c|c|c|c|c|c|}
\hline Clon & Altura Planta (cm) & Posición Frutos & Diámetro Follaje (cm) & Forma fruto & Forma hoja & Arq. Planta & $\mathrm{N}^{\circ}$ frutos \\
\hline C-01 & 140 & A & 181 & $A-\mathrm{m}$ & $\mathrm{E}-\mathrm{m}$ & MB & 592 \\
\hline C-02 & 92 & C & 15 & $A-m$ & $\mathrm{E}-\mathrm{m}$ & $\mathrm{B}$ & 464 \\
\hline C-03 & 63 & $\mathrm{C}$ & 91 & $\Lambda-p$ & E-g & $\mathrm{R}$ & 408 \\
\hline C-04 & 45 & $\mathrm{C}$ & 65 & $A-p$ & E-g & B & 496 \\
\hline C-05 & 89 & C & 81 & $A-m$ & E-m & B & 1072 \\
\hline C-07 & 69 & C & 73 & E-p & E-p & B & 656 \\
\hline C-08 & 69 & A & 115 & E-p & E-g & B & 1200 \\
\hline C-09 & 101 & A & 85 & E-p & E-m & B & 224 \\
\hline$C-10$ & 59 & A & 90 & E-p & E-m & B & 1136 \\
\hline C-11 & 91 & C & 113 & E-p & E-p & $\mathrm{MB}$ & 4128 \\
\hline C-12 & 242 & C & 234 & E-p & E-p & $\mathrm{MB}$ & 4544 \\
\hline C-13 & 54 & $A$ & 51 & R-p & E-p & B & 1328 \\
\hline C-14 & 19 & C & 82 & A-p & E-m & B & 560 \\
\hline C-15 & 172 & A & 134 & E-p & E-p & B & 2400 \\
\hline C-16 & 57 & C & 94 & E-p & E-p & B & 1760 \\
\hline C-17 & 82 & C & 107 & E-p & E-m & B & 1264 \\
\hline C-18 & 56 & C & 110 & R-p & $\mathrm{E}-\mathrm{m}$ & B & 2160 \\
\hline C-19 & 67 & C & 91 & E-p & $\mathrm{E}_{-\mathrm{g}}$ & $B$ & 1440 \\
\hline C-20 & 63 & A & 99 & E-p & E-m & B & 2144 \\
\hline C-21 & 112 & A & 124 & E-m & E-g & B & 2192 \\
\hline C-22 & 74 & A & 141 & E-m & E-m & B & 1136 \\
\hline C-23 & 66 & C & 107 & E-m & E-g & B & 1120 \\
\hline C-24 & 77 & C & 99 & E-m & E-g & B & 528 \\
\hline C-25 & 87 & C & 131 & E-m & E-g & B & 768 \\
\hline C-27 & 128 & $\mathrm{C}$ & 154 & $\mathrm{R}-\mathrm{m}$ & E-g & B & 1152 \\
\hline C-28 & 61 & C & 92 & E-m & $E_{-g}$ & B & 480 \\
\hline$C-30$ & 143 & C & 145 & E-p & $\mathrm{E}-\mathrm{m}$ & B & 2592 \\
\hline C-31 & 76 & C & 121 & E-p & $A-m$ & B & 1776 \\
\hline C-32 & 134 & A & 192 & E-m & E-p & B & 3328 \\
\hline C-33 & 154 & A & 223 & E-m & E-m & B & 2688 \\
\hline C-34 & 83 & C & 126 & E-p & E-p & B & 1696 \\
\hline C-35 & 117 & A & 194 & E-m & E-g & MB & 2400 \\
\hline C-36 & 83 & A & 162 & E-g & E-m & $\mathrm{MB}$ & 2720 \\
\hline$C-37$ & 82 & $A$ & 141 & $\mathrm{R}_{-\mathrm{p}}$ & E-m & B & 1232 \\
\hline C-38 & 87 & A & 172 & E-m & E-m & MB & 2816 \\
\hline C-39 & 67 & A & 94 & R-p & E-p & B & 800 \\
\hline C-40 & 89 & A & 155 & E-m & E-m & MB & 2640 \\
\hline C-41 & 126 & C & 147 & E-m & E-m & B & 3088 \\
\hline C-42 & 110 & $A$ & 236 & $\mathrm{R}-\mathrm{m}$ & E-m & MB & 4160 \\
\hline$C-43$ & 113 & $A$ & 209 & R-p & $\mathrm{E}-\mathrm{m}$ & MB & 3312 \\
\hline C-44 & 111 & C & 161 & E-m & E-m & B & 1888 \\
\hline$C-45$ & 67 & A & 102 & R-m & $\mathrm{O}-\mathrm{p}$ & B & 1520 \\
\hline C-46 & 75 & A & 100 & E-m & A-m & B & 128 \\
\hline$C-47$ & 81 & A & 91 & E-m & E-m & B & 192 \\
\hline C-48 & 170 & $A$ & 170 & R-m & $\mathrm{O}-\mathrm{m}$ & MB & 2496 \\
\hline C-49 & 76 & C & 81 & E-m & $\Lambda-p$ & B & 576 \\
\hline C-50 & 49 & $A$ & 63 & R-m & $\mathrm{O}-\mathrm{m}$ & $\mathrm{R}$ & 800 \\
\hline C-51 & 114 & A & 168 & E-m & E-m & $\mathrm{MB}$ & 2512 \\
\hline C.53 & 112 & C & 154 & R-p & A-m & MB & 3360 \\
\hline C-54 & 82 & C & 118 & E-g & $\mathrm{O}-\mathrm{m}$ & B & 3248 \\
\hline
\end{tabular}

Posición de frutos $\mathrm{A}=$ Alterno, $\mathrm{C}=$ Continuo, $\mathrm{R}=\mathrm{Racimo}$, Diámetro de follaje $(\mathrm{cm})$ Forma de fruto, $\mathrm{A}-\mathrm{p}=\mathrm{Alargado}$ pequeño, $\mathrm{A}-\mathrm{m}=\mathrm{Alargado}$ mediano, A-g = Alargado grande, E-p= Elíptica pequeño $\mathrm{E}, \mathrm{m}=$ Elíptica mediana, E-g= Elíptica grande, $\mathrm{R}-\mathrm{p}=\mathrm{Redonda}$ pequeño, $\mathrm{R}-\mathrm{m}=\mathrm{Redonda}$ mediana, $\mathrm{R}-\mathrm{g}=$ Redonda grande, Forma de hoja, E-p= Elíptica pequeño, E-m= Elíptica mediana, E-g=Eliptica grande, $\mathrm{A}-\mathrm{p}=\mathrm{Alargado}$ pequeño, $\mathrm{A}$ - $\mathrm{m}=$ Alargado mediano, $\mathrm{A}-\mathrm{g}=\mathrm{Alargado}$ grande, $\mathrm{O}-\mathrm{p}=$ Ovalada pequeño, $\mathrm{O}-\mathrm{m}=$ Ovalada mediana, $\mathrm{O}-\mathrm{g}=\mathrm{Ovalada}$ grande, Arquitectura de planta $\mathrm{R}=\mathrm{Planta}$ de regular arquitectura, $\mathrm{B}=$ Planta de buena arquitectura, $\mathrm{MB}=$ Planta de muy buena arquitectura Número de frutos. Se contó el total de frutos por clon. 
Arévalo, N. Efecto de biofertilizante y materia orgánica en el rendimiento de clones de jojoba en los Pichones Tacna 2014.

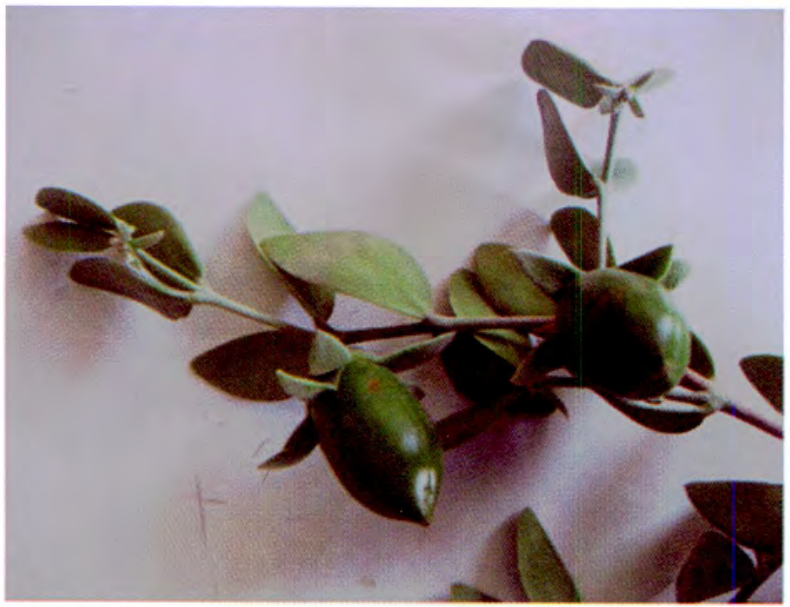

Figura 5. Fructificación alterna.

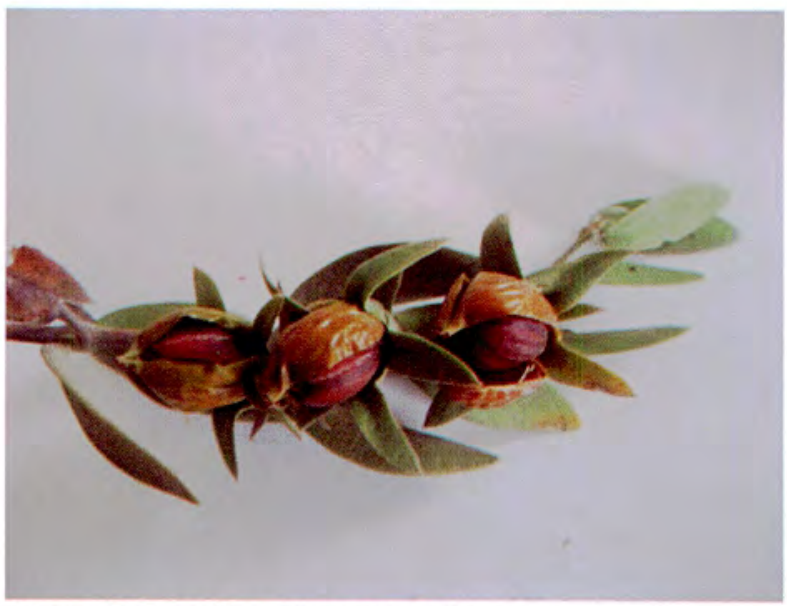

Figura 7. Fructificación continua (frutos maduros).

Tabla 3. Prueba de Duncan del peso (g) de clones de jojoba con aplicación de biofertilizantes y materia orgánica. Los Pichones-Tacna 2014.

\begin{tabular}{ccc}
\hline Tratamiento & Promedio $(\mathrm{g})$ & Nivel de significación \\
\hline T5 & 784,40 & $\mathrm{~A}$ \\
T4 & 758,80 & $\mathrm{~A}$ \\
T3 & 636,00 & $\mathrm{~B}$ \\
T2 & 484,40 & $\mathrm{C}$ \\
T1 & 393,40 & $\mathrm{C}$ \\
\hline
\end{tabular}

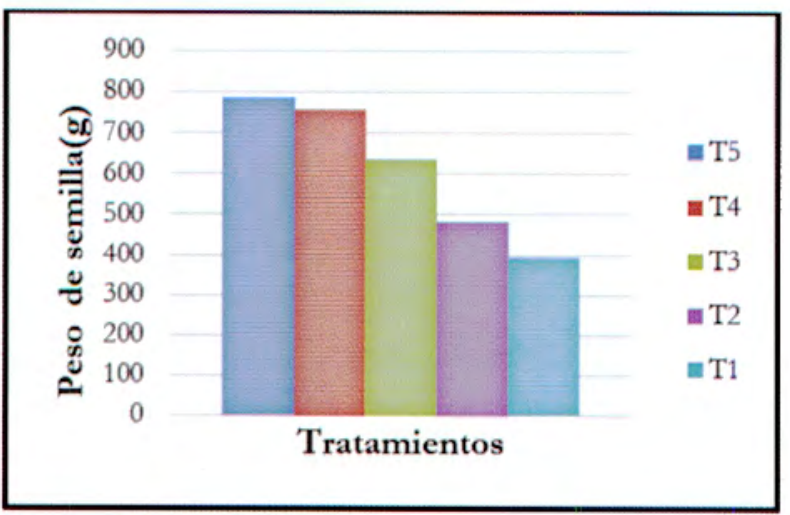

Figura 9. Efecto de la biofertilización y materia orgánica en el peso de semillas de clones de jojoba, Los Pichones, Tacna-2014.

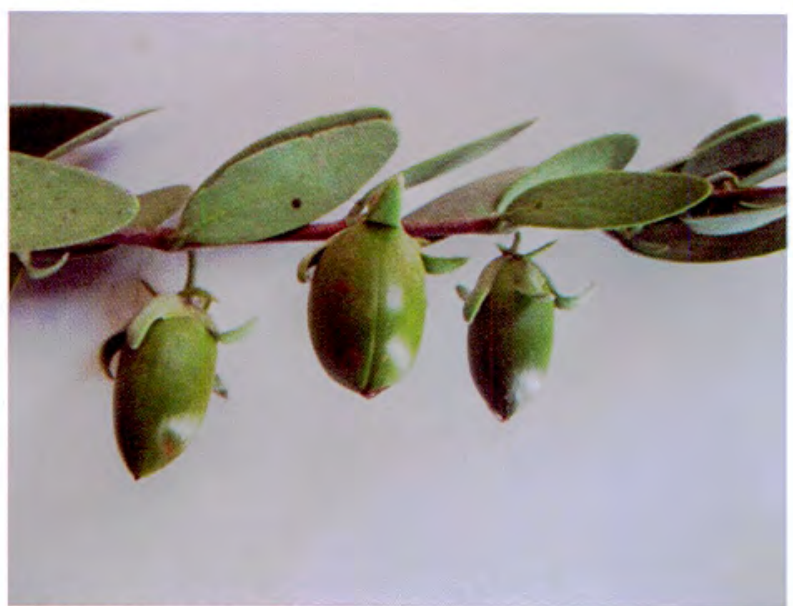

Figura 6. Fructificación continua.

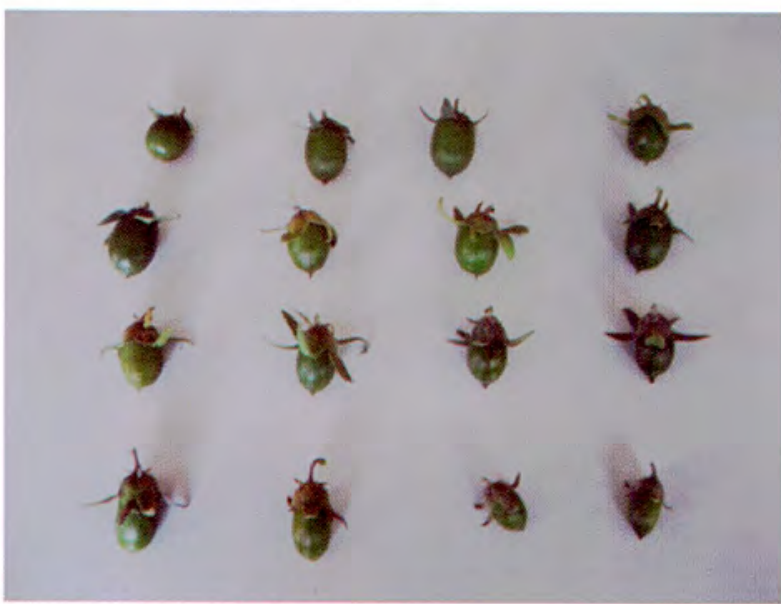

Figura 8. Forma de frutos.

Tabla 4. Prueba de Duncan del Rendimiento $\mathrm{kg} / \mathrm{ha}$ de clones de jojoba con aplicación de biofertilizantes y materia orgánica. Los Pichones-Tacna 2014.

\begin{tabular}{ccc}
\hline Tratamiento & Promedio $(\mathrm{kg} / \mathrm{ha})$ & Nivel de significación \\
\hline T5 & 2353,20 & $\mathrm{~A}$ \\
T4 & 2276,40 & $\mathrm{~A}$ \\
T3 & 1908,00 & $\mathrm{~B}$ \\
T2 & 1453.20 & $\mathrm{C}$ \\
T1 & 1180,20 & $\mathrm{C}$ \\
\hline
\end{tabular}

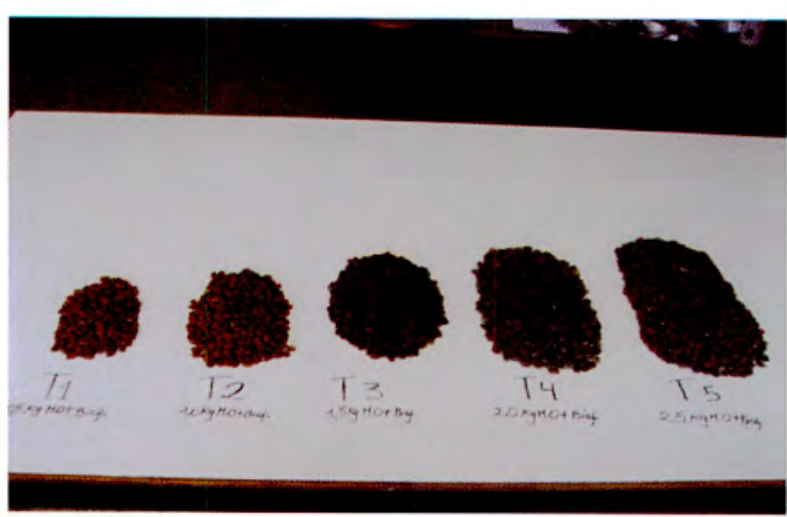

Figura 10. Peso de semillas con tratamientos de M.O. y biofertilizante. 
Existe diferencias en el peso de semillas (g) de clones de jojoba entre los tratamientos, el mayor peso se obtuvo con el T5 $(784,40 \mathrm{~g})$ seguido del T4 con $758,80 \mathrm{~g}$ que no difieren estadisticamente entre ellos. Siendo el T1 con 393,40 g que tiene el promedio más bajo. (Tabla 3, figura 9 y figura 10).

Existe diferencias en el Rendimiento $\mathrm{kg} / \mathrm{ha}$ de clones de jojoba entre los tratamientos, el mayor rendimiento se obruvo con el T5 (2 353,20 kg/ha) seguido del T4 con 2 $276,40 \mathrm{~kg} / \mathrm{ha}$ que no difieren estadísticamente entre ellos. Siendo el T1 con $1180,20 \mathrm{~kg} / \mathrm{ha}$ que tiene el promedio más bajo. (Tabla 4).

\section{DISCUSIÓN}

El nutriente del suelo que requiere un vegetal en mayor cantidad es el nitrógeno, ya que influye en el incremento de biomasa, sin embargo a pesar de su función crítica en la nutrición vegetal, el nitrógeno es asimilado así completamente en estado inorgánico en forma de nitrato o amonio (Alexander, 1980). La conversión del nitrógeno orgánico al estado inorgánico más móvil se conoce como mineralización de nitrógeno. Como consecuencia en la mineralización se produce amonio y nitrato y desaparece el nitrógeno orgánico, estos productos delimitan dos procesos microbiológicos distintos: amonificación, en donde el amonio se forma a partir de compuestos orgánicos y la nitrificación, término que usualmente se adopta para referirse a la oxidación del amonio a nitrato.

Martins, Angers y Corá (2012), mencionan la necesidad de trabajar en el manejo ecológico del suelo como una herramienta importante de la agricultura orgánica y dentro de esta tarea se busca actuar sobre las parcelas orgánicas de tal forma que permitan un aumento del contenido de la materia orgánica, lo cual a su vez tendría un efecto positivo sobre la biología del suelo, asimismo han señalado que para reducir las pérdidas y facilitar el uso óptimo del nitrógeno mineralizado por el cultivo en crecimiento, es necesario conocer el efecto del manejo de los predios agrícolas sobre los organismos del suelo y del ciclo del nitrógeno. Además, estos efectos ayudarían a reducir los problemas ambientales porque permitirían una reducción considerable de la fertilización nitrogenada, debido a una alta mineralización del nitrógeno desde la materia orgánica.

Los biofertilizantes son preparados que contienen células microbianas vivas o latentes que se usan para mejorar la fertilidad del suelo como las bacterias fijadoras del nitrógeno, solubilizadoras de fósforo, potencializadoras de diversos nutrientes o productos de sustancias activas. Estos biopreparados son capaces de suministrar a los cultivos entre $15 \%$ y $50 \%$ de sus necesidades de nitrógeno mediante su capacidad de captación del nitrógeno atmosférico, además, su capacidad para sintetizar sustancias biológicamente activas permiten acortar los ciclos de cultivo e incrementar los rendimientos entre $30 \%$ y $50 \%$ (Alarcón et al., 2009).

Los tratamientos que recibieron biofertilizante $(A z 0-$ tobacter sp.) y mayor dosis de materia orgánica (T5=biofertilizante $+2,5 \mathrm{~kg} /$ planta de M.O y T4=biofertilizante $+2,0 \mathrm{~kg} /$ planta de M.O) lograron los mayores rendimientos, pudiendo afirmar que los tratamien- tos orgánicos son considerados comó una buena alternativa para mejorar la características físicas, químicas y microbiológicas del suelo y mantener una producción orgánica sostenible como lo sostienen Borda et al. (2011), en comparación a la aplicación con tratamicnto con fertilizante químicos por sus efectos negativos en las características del suelo y los altos costos de los fertilizantes químicos entre otros.

Pellicer et al. (2008) y Rodriguez et al. (2010) trabajaron con pimiento y aji jalapeño respectivamente, combinando fertilización química, fertilización orgánica y biofertilización logrando un incremento en el número de frutos y peso por planta superior a los tratamientos con solo fertilización química.

Asimismo Alarcón et al. (2009) señalan que los mejores rendimientos de tomate se alcanzaron aplicando altas concentraciones de Azotobacter.

Los incrementos en rendimiento obtenidos en esta investigación se alcanzaron aplicando mayor cantidad de materia orgánica $(2,5 \mathrm{~kg} /$ planta) siendo estos resultados similares a los reportados por estos autores.

\section{CONCLUSIONES}

La aplicación de biofertilizante y materia orgánica tienen efectos positivos en las características morfológicas y el rendimiento de clones de jojoba en el fundo Los Pichones.

I os caracteres altura de planta, número de frutos se modificaron con la aplicación de biofertilizante y materia orgánica.

Los caracteres forma de hoja, de frutos y posición de frutos no se modificaron con la aplicación de biofertilizante y materia orgánica.

Con aplicación de biofertilizante más $2,5 \mathrm{~kg} /$ planta de materia orgánica se obtuvo un peso de semilla de 784,40 gramos/ planta superando al testigo (T1) que tuvo un rendimiento de 393,40 gramos/planta.

Con aplicación de biofertilizante más $2,5 \mathrm{~kg} /$ planta de materia orgánica se obtuvo un rendimiento de 2353,20 $\mathrm{kg} / \mathrm{ha}$ superando al testigo (T1) que tuvo un rendimiento de $1180,20 \mathrm{~kg} / \mathrm{ha}$.

\section{RECOMENDACIONES}

Teniendo en cuenta que debemos conservar el medio ambiente con un desarrollo sostenible, se recomienda:

- Efectuar investigaciones con aplicación de biofertilizantes y productos orgánicos (abonos orgánicos, biocidas, rotación de cultivos, alelopatía entre otros) en diferentes cultivos, teniendo en cuenta que Tacna es una zona desértica con problemas de salinidad y escasez de recurso hídrico para evitar la contaminación de los suclos, acuifero, la flora y la fauna por el uso de fertilizantes sintéticos.

- Efectuar investigaciones con aplicación de biofertilizantes y otras fuentes orgánicas como humus de lombriz, abonos verdes entre otros, con dosis y momentos de aplicación para evaluar sus efectos y sostenibilidad sobre el rendimiento de jojoba.

- Fomentar como línea de investigación en la Escuela de 
Arévalo, N. Efecto de biofertilizante y materia orgánica en el rendimiento de clones de jojoba en los Pichones Tacna 2014.

Agronomía de la Facultad de Ciencias Agropecuarias empleando sistemas de cultivo como policultivo y rotacional, además con el uso de productos orgánicos y biofertilizantes tendiente a una cultura orgánica.

\section{REFERENCIAS BIBLIOGRÁFICAS}

Alarcón, A., Alarcón, A., Godefoy, M., Gonzales, G. (2009). Efecto de diferentes concentraciones de Azotobacter cbroococcum sobre algunos parámetros de crecimiento y el rendimiento del tomate (Lycopersicum esculentum, Mill). Cuba. Revista Electrónica Granma Ciencia. vol. 13 (1), Enero-Abril.

Alexander, M. (1980). Introducción a la Microbiologia del Suelo 2daEd. México: AGT, 491 pp.

Araujo Y. Diaz L. Rodriguez F., Pargas L., (2010). Efecto del biofertilizante Azotobacter sp. en el cultivo de papa en el estado Mérida. Instituto Nacional de Investigaciones Agrícolas del Estado Mérida. Mérida, Venezuela, Resumen 6 p.

Arévalo, N. y Fernandez, O. (2011). Mejoramiento genético y adaptación de genotipos y clones superiores de jojoba Sinmondsia cbinensis Link Scbneider en zonas áridas salinas de Tacna. Revista Ciencia \& Desarrollo, vol. 13.

Arévalo, N. (2014) Efecto de la materia orgánica y el Azotobacter sp. en el suelo y el rendimiento de algodón de color Gossypium barbadense L. en el fundo Los Pichones Tacna- 2013. Tesis Universidad Nacional Jorge Basadre Grohmann Escuela de Posgrado. Tacna.

Borda, D., Pardo, J., Martinez, M., Montaña, J. (2011). Producción de un Biofertilizante a partir d un aislamiento de Azotobacter nigricans obtenido en un cultivo de Stevia rebaudiana Bert. Univ. Sci. vol.14 (1). Bogotá enero/abril.

Pellicer, C., Pérez, A., Abadia, A., Rincón, L., Paredes, A., Carrillo, F (2008). Resultado del aporte de biofertilizante a un cultivo de pimiento con fertilización ecológica. Ponencia presentado VIII. Congreso SEAE, IV Congreso Iberoamericano agroecología. BullasMurcia.

Rodríguez E., Bolaños M, Menjivar, J. (2010). Efecto de la fertilización en la nutrición y rendimiento de ají (Capsicum sp.) en el Valle del Cauca, Colombia Esp. Cipag 10 Acta agronómica. 59 (1), p 55-64.
Correspondencia:
Nelly Arévalo Solsol: arevalonelly@hotmail.com
Fecha de Recepción: 23/03/2015

Fecha de Aceptación: 02/06/2015 